\title{
Article
}

\section{(S)-Reutericyclin: Susceptibility Testing and In Vivo Effect on Murine Fecal Microbiome and Volatile Organic Compounds}

\author{
Bernhard Kienesberger ${ }^{1}$, Beate Obermüller ${ }^{1, *}$, Georg Singer ${ }^{1}\left(\mathbb{D}\right.$, Barbara Mittl ${ }^{1}$, Reingard Grabherr ${ }^{2}{ }^{\oplus}$, \\ Sigrid Mayrhofer ${ }^{2}$, Stefan Heinl ${ }^{2}$, Vanessa Stadlbauer ${ }^{3}{ }^{(0}$, Angela Horvath ${ }^{3,4}{ }^{(0)}$, Wolfram Miekisch ${ }^{5}(\mathbb{0}$, \\ Patricia Fuchs ${ }^{5}{ }^{\infty}$, Ingeborg Klymiuk ${ }^{6}{ }^{\oplus}$, Holger Till $^{1}$ and Christoph Castellani ${ }^{1}{ }^{\circledR}$
}

check for

updates

Citation: Kienesberger, B. Obermüller, B.; Singer, G.; Mittl, B.; Grabherr, R.; Mayrhofer, S.; Heinl, S.; Stadlbauer, V.; Horvath, A.; Miekisch, W.; et al. (S)-Reutericyclin: Susceptibility Testing and In Vivo Effect on Murine Fecal Microbiome and Volatile Organic Compounds. Int. J. Mol. Sci. 2021, 22, 6424. https:// doi.org/10.3390/ijms22126424

Academic Editor: Silvia Melgar

Received: 20 May 2021

Accepted: 14 June 2021

Published: 15 June 2021

Publisher's Note: MDPI stays neutral with regard to jurisdictional claims in published maps and institutional affiliations.

Copyright: (C) 2021 by the authors. Licensee MDPI, Basel, Switzerland. This article is an open access article distributed under the terms and conditions of the Creative Commons Attribution (CC BY) license (https:// creativecommons.org/licenses/by/ $4.0 /)$.
1 Department of Paediatric and Adolescent Surgery, Medical University of Graz, 8036 Graz, Austria; bernhard.kienesberger@medunigraz.at (B.K.); georg.singer@medunigraz.at (G.S.); barbara.mittl@medunigraz.at (B.M.); holger.till@medunigraz.at (H.T.); christoph.castellani@medunigraz.at (C.C.)

2 Department of Biotechnology, University of Natural Resources and Life Sciences Vienna, 1190 Vienna, Austria; reingard.grabherr@boku.ac.at (R.G.); sigrid.mayrhofer@boku.ac.at (S.M.); stefan.heinl@boku.ac.at (S.H.)

3 Department of Internal Medicine, Division of Gastroenterology and Hepatology, Medical University of Graz, 8036 Graz, Austria; vanessa.stadlbauer@medunigraz.at (V.S.); angela.horvath@medunigraz.at (A.H.)

4 Center of Biomarker Research (CBmed), 8036 Graz, Austria

5 Experimental Research Center, Department of Anesthesiology and Intensive Care, Rostock University Medical Center, 18057 Rostock, Germany; wolfram.miekisch@uni-rostock.de (W.M.); patricia.fuchs@uni-rostock.de (P.F.)

6 Gottfried Schatz Research Center, Department of Cell Biology, Histology and Embryology, Medical University of Graz, 8036 Graz, Austria; ingeborg.klymiuk@medunigraz.at

* Correspondence: beate.obermueller@medunigraz.at; Tel.: +43-316-385-13762

Abstract: We aimed to assess the in vitro antimicrobial activity and the in vivo effect on the murine fecal microbiome and volatile organic compound (VOC) profile of (S)-reutericyclin. The antimicrobial activity of (S)-reutericyclin was tested against Clostridium difficile, Listeria monocytogenes, Escherichia coli, Enterococcus faecium, Staphylococcus aureus, Staphylococcus (S.) epidermidis, Streptococcus agalactiae, Pseudomonas aeruginosa and Propionibacterium acnes. Reutericyclin or water were gavage fed to male BALBc mice for 7 weeks. Thereafter stool samples underwent $16 \mathrm{~S}$ based microbiome analysis and VOC analysis by gas chromatography mass spectrometry (GC-MS). (S)-reutericyclin inhibited growth of S. epidermidis only. Oral (S)-reutericyclin treatment caused a trend towards reduced alpha diversity. Beta diversity was significantly influenced by reutericyclin. Linear discriminant analysis Effect Size (LEfSe) analysis showed an increase of Streptococcus and Muribaculum as well as a decrease of butyrate producing Ruminoclostridium, Roseburia and Eubacterium in the reutericyclin group. VOC analysis revealed significant increases of pentane and heptane and decreases of 2,3-butanedione and 2heptanone in reutericyclin animals. The antimicrobial activity of (S)-reutericyclin differs from reports of (R)-reutericyclin with inhibitory effects on a multitude of Gram-positive bacteria reported in the literature. In vivo (S)-reutericyclin treatment led to a microbiome shift towards dysbiosis and distinct alterations of the fecal VOC profile.

Keywords: reutericyclin; bacteriocin; microbiome; antimicrobial activity; VOC; isoform; antibiotic; resistance

\section{Introduction}

The importance of the intestinal microbiome has gained wide scientific interest in both health and disease. A plethora of factors including nutritional habits, medication or chronic illnesses influences the microbiome composition. Many diseases such as type II diabetes, obesity, cancer cachexia, chronic cardiovascular or chronic inflammatory bowel disease have been associated with dysbiosis-an alteration of the composition of the microbiome with negative effects on the host [1-5]. Therefore, research has focused on possibilities to 
shift the intestinal microbiome towards a "healthier" composition as a possible therapeutic or supportive approach. Apart from dietary modifications, antibiotics or stool transplantations, this could be achieved by nutritional supplementation with either pre-, pro-, syn- or postbiotics. While probiotics are generally considered as safe, infections with probiotics may occur in vulnerable patient cohorts on rare occasions. Therefore, alternatives to live microorganisms to manage dysbiosis are of interest [6].

Some intestinal bacteria are able to produce specific substances inhibiting growth or inactivating other competitive strains in order to defend their own habitat. This bacterial defensive strategy is based on different classes of antimicrobial compounds. Some antimicrobial metabolites like acetic acid, lactic acid, or reuterin are small organic compounds $[7,8]$. Others like reutericin are short, ribosomally synthesized, post-translationally modified peptides termed bacteriocins $[9,10]$. Finally, reutericyclin is another form of antimicrobial substance synthesized by a non-ribosomal peptide synthetase [11]. Due to their antimicrobial effects, all these substances, which are also contained in postbiotics, may be attractive alternatives to treat dysbiosis, such as in immunocompromised patients.

In 2000, reutericyclin (4-acetyl-1-[(E)-dec-2-enoyl]-3-hydroxy-2-(2methylpropyl)-2Hpyrrol-5-one) was first mentioned as a low molecular weight antibiotic produced by certain lactic acid bacteria [12]. Reutericyclin, with a molecular mass of $349 \mathrm{Da}$, is a typical 1,3bisacyltetramic acid and consists of four methyl and seven methylene groups, as well as two aliphatic and two olefinic methine groups, as shown in the corresponding HSQC spectrum [13,14]. With this structure, reutericyclin is an amphiphilic molecule consisting of a hydrophilic, negatively charged head group with two hydrophobic side chains [15]. It is a weak acid and its activity is increased at low $\mathrm{pH}$ levels [14]. Reutericyclin exists in (R)- and (S)-isoforms at $C_{5}$. Reutericyclin was found to inhibit growth of many Grampositive bacteria [16]. This bacteriocidal effect of reutericyclin has been attributed to its action as a proton ionophore [15]. In this way, it selectively dissipates the bacterial transmembrane potential [17,18]. Fungi, yeasts and gram-negative bacteria, however, have been described as being resistant to reutericyclin [15]. Reutericyclin producing strains of Limosilactobacillus ( $L$.) reuteri are broadly utilized in food fermentations [12] and have also been previously described as a stable constituent of the intestinal microbiota of both humans and animals [19]. Therefore, the application of reutericyclin appears to be a save approach. However, at present there are only limited reports regarding the effect of reutericyclin on the intestinal microbiome in vivo. While there is no such study on reutericyclin as pure substance, there are a few reports investigating the effect of reutericyclin producing probiotic $L$. reuteri in piglets $[20,21]$. In one study, this decreased the abundance of the L. reuteri group in fecal samples in comparison to controls [21]. These results suggest that reutericyclin is a subtle but significant modulator of the Lactobacillus community in pigs [21]. In contrast, Wang et al. report that the probiotic derived antimicrobial compound reutericyclin had only a limited impact on swine intestinal microbiota [20].

The aims of this study were to examine (1) the in vitro antimicrobial activity of (S)reutericyclin, (2) its in vivo effect as pure substance on the fecal microbiome, and (3) the fecal volatile organic compound (VOC) profile in a murine model.

\section{Results}

\subsection{Susceptibility Testing}

(S)-reutericyclin exhibited antimicrobial activity against Staphylococcus (S.) epidermidis only. Neither cooking, buffering, nor treatment with $\mathrm{HCl}$ or proteinase $\mathrm{K}$ influenced this activity (Figure 1). 


\section{S. epidermidis}

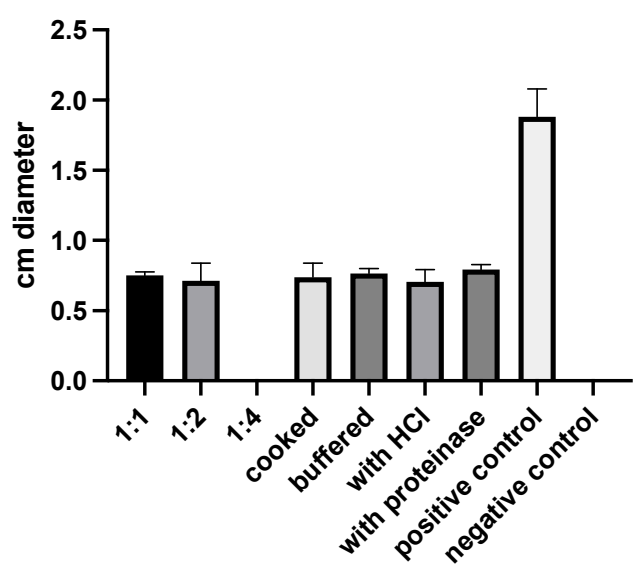

Figure 1. Results of susceptibility testing with (S)-reutericyclin.

\subsection{In Vivo Microbiome Analysis}

The microbiome analysis revealed a trend towards reduced alpha diversity and significantly reduced Bray-Curtis dissimilarity in animals receiving (S)-reutericyclin (Figure 2).
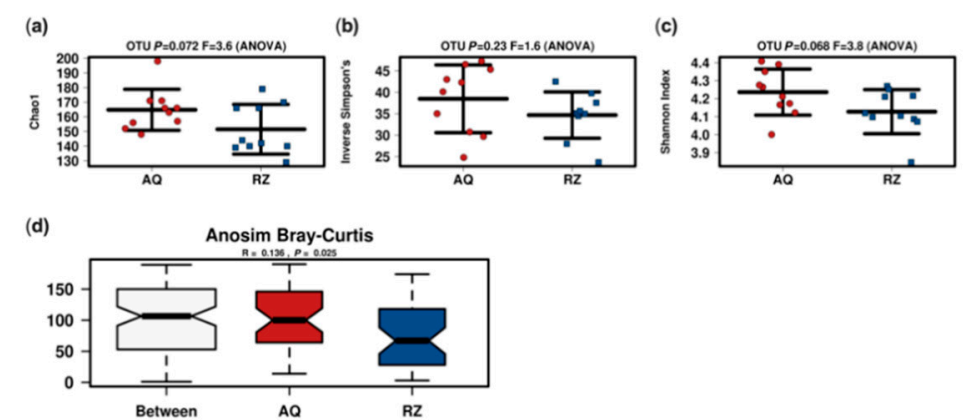

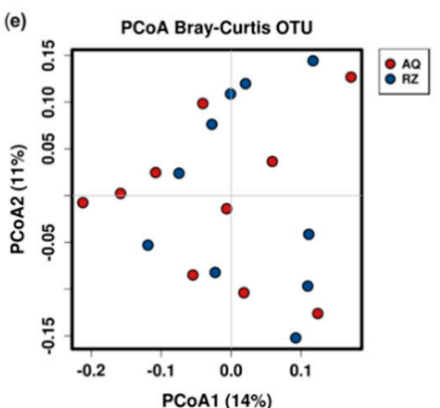

(g)

$A Q$

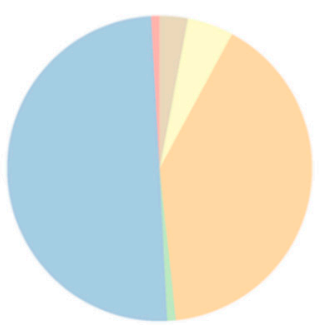

(f)

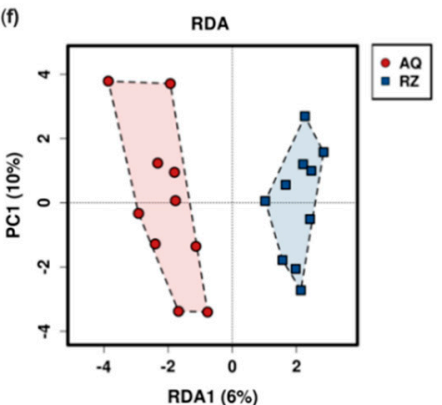

$\mathbf{R Z}$

Figure 2. Microbiome analysis of the two groups. Alpha $(\mathbf{a}-\mathbf{c})$ and beta-diversity; $(\mathbf{d}-\mathbf{f})$ indices (RDA: variance 23.12; F 1.17; $p$ 0.07); pie charts of the mean relative abundance at the phylum level (g). $\mathrm{AQ} \ldots$ aqua control group; RZ ... (S)-reutericyclin group. 
The linear discriminant analysis effect size (LEfSe) analysis at the species level between the two groups showed significant differences in mice undergoing (S)-reutericyclin treatment compared to the control group (Figure 3).

(a)

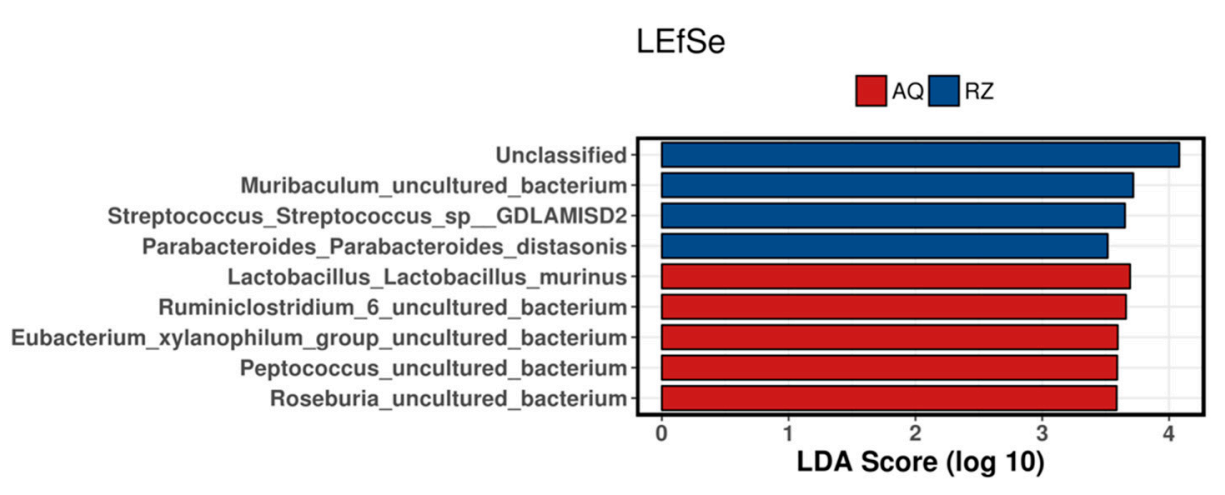

(b)

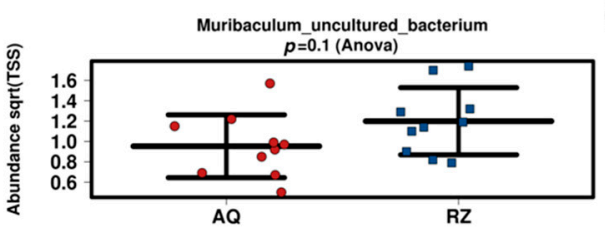

(d)

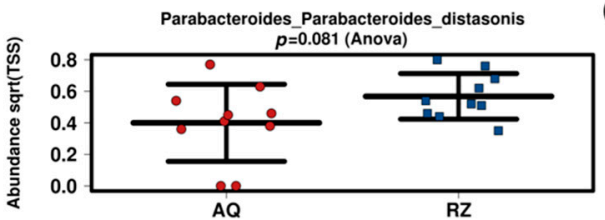

(f)

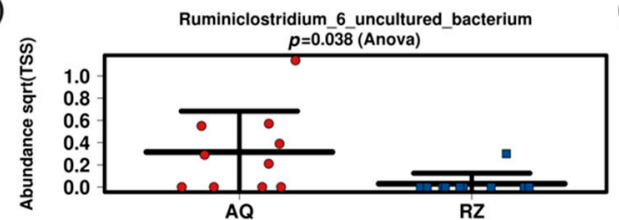

(h)

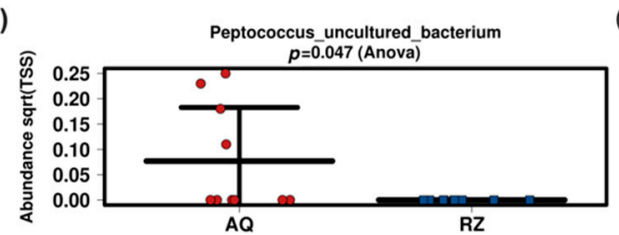

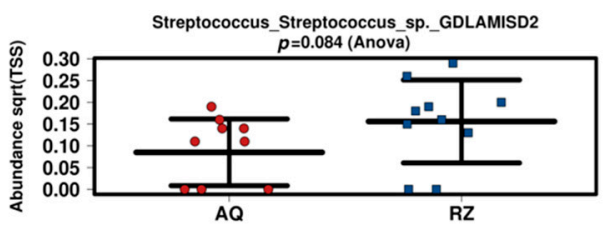

(e)

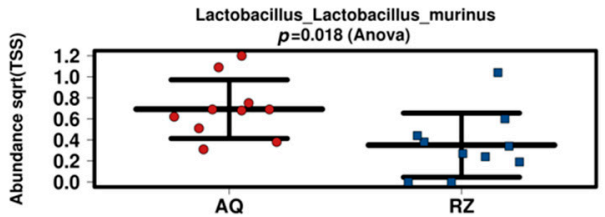

(g)

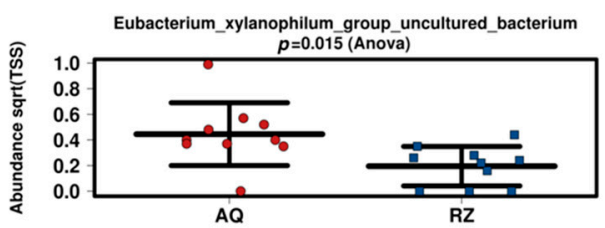

(i)

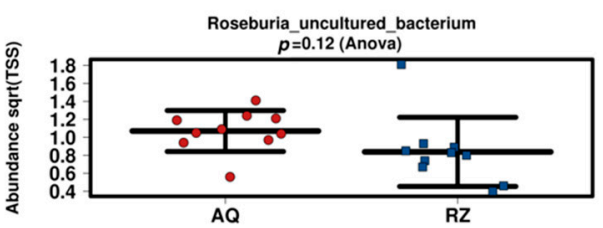

Figure 3. Linear discriminant (LDA) and effect size (LEfSe) analysis of the two groups (a). Strip charts (mean and standard deviation) of the bacteria altered in the LEfSe analysis (b-i). AQ-aqua control group; RZ-(S)-reutericyclin group.

\subsection{Fecal Volatile Organic Compound Analysis}

A total of 42 VOCs could be detected tentatively in the headspace of murine stool samples. The mean stool sample weight was $25 \mathrm{mg}$ (SD $3.4 \mathrm{mg}$ ). The alterations of four substances (propene, isopropyl alcohol, isoflurane and o-xylene) could be attributed to room air contamination. Another five substances (propanol, acetic acid, 2-methylpentane, 3-methylpentane and butanoic acid butyl ester) were detected in $<2$ samples and were thus excluded from the further analysis. This left 33 candidate substances for statistical comparison. Pentane and heptane were significantly increased, and 2,3-butanedione and 2-heptanone were significantly decreased in animals of the (S)-reutericyclin group. Butanoic acid propyl ester showed a trend towards an increase and (z)-2-butene and acetoacetate methyl ester towards a decrease in mice gavage fed with (S)-reutericyclin. 
Dendrogram analysis revealed a clustering within ketones, esters and short chained carbohydrates (Figure 4).

(a)

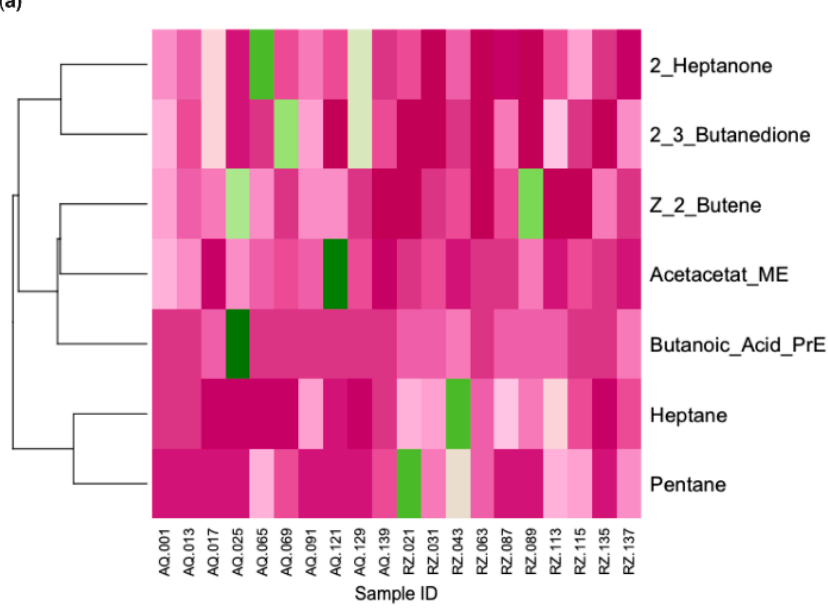

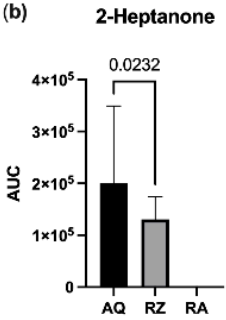

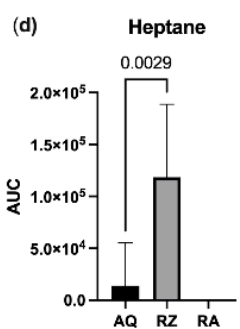

(c) 23-Butanedione

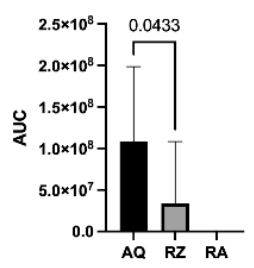

(e)

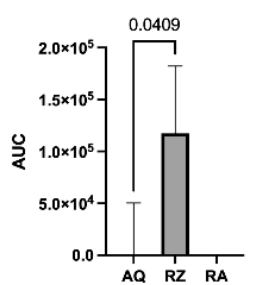

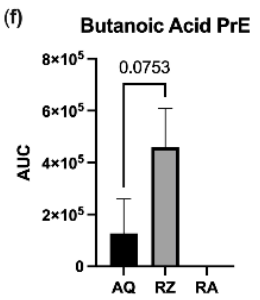
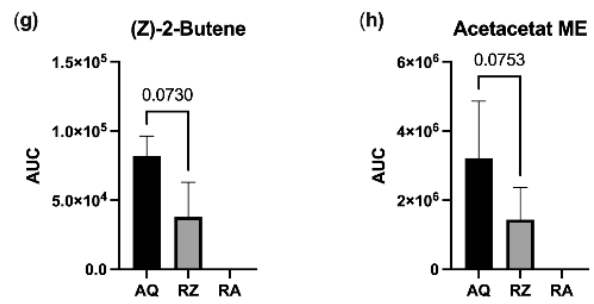

Figure 4. Results of the VOC analysis. (a) Heatmap with dendrogram. VOC values were normalized to the maximum; colors range from dark green $(=1.0)$ to dark pink $(=0.0)$; substances with significant changes $(\mathbf{b}-\mathbf{e})$ and with a trend between (f-h) the groups. AQ ... aqua control group; RZ . . (S)-reutericyclin group; RA . . room air samples; PrE ... propyl ester; ME ... methyl ester.

\subsection{Correlation Analysis}

The results of the Spearman-Rho correlation analysis between the bacteria altered in LEfSe analysis and VOCs with a group difference $p<0.1$ are shown in Figure 5.

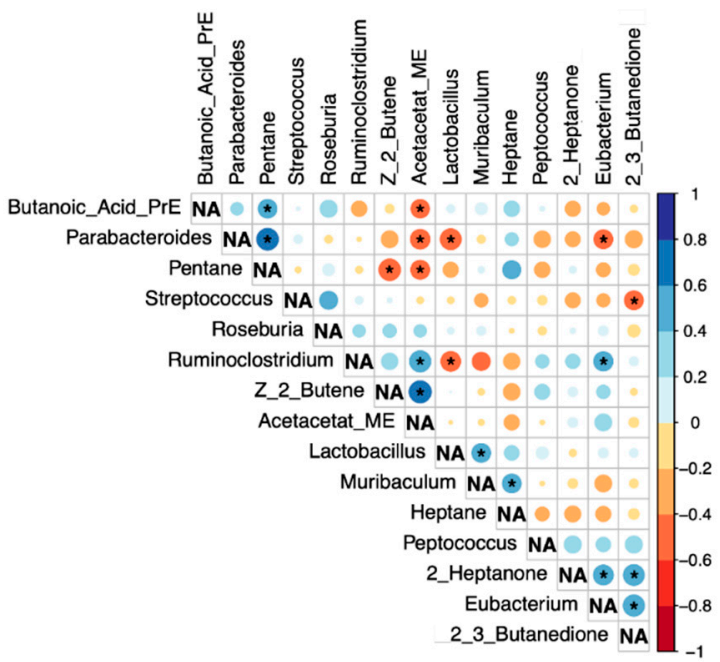

Figure 5. Correlation analysis (Spearman-Rho). Significant correlations $(p<0.05)$ are marked with * NA ... not applicable. 


\section{Discussion}

In this investigation we tested the in vitro antimicrobial activity of (S)-reutericyclin and found a different reactivity than expected from the (R)-isoform reported in the literature. Furthermore, we gavage fed mice with (S)-reutericyclin and detected a shift in the microbiome towards dysbiosis compared to control animals. Finally, (S)-reutericyclin lead to distinct alterations of the fecal VOC profile as a marker for the bacterial metabolism.

In our in vitro susceptibility testing (S)-reutericyclin exhibited antimicrobial effects against Staphylococcus (S.) epidermidis, but not against the other bacteria tested. While the lack of an effect on Gram-negative bacteria (Escherichia (E.) coli, Campylobacter (C.) jejuni and Pseudomonas (P.) aeruginosa) was expected, a reactivity against the other Gram-positive bacteria should have been detectable [15]. Our finding is in sharp contrast to examinations by Hurdle et al. who described excellent potency of reutericyclins ((R)-reutericyclin and chemical modifications) against the lethal non-growing stage of Clostridium (C.) difficile at concentrations that may also be attained in the gastrointestinal tract [17]. In their study, they describe a mean minimal inhibitory concentration (MIC) of 0.09-0.5 mg/L depending on the strain [17]. Although we applied (S)-reutericyclin at a concentration of $62.5 \mu \mathrm{g} / \mathrm{mL}$ (resulting in $1.25 \mu \mathrm{g} /$ platelet for the 1:1 concentration), we could not achieve an inhibitory effect. One reason for this finding may lie in the different culture methods. Hurdle et al. used TY broth, while we cultured on plates with tryptic soy broth supplemented with sheep blood. As reutericyclin showed activity against S. epidermidis, it obviously migrated into the culture medium. However, it may be possible that the final concentration in the agar was too low to inhibit $C$. difficile in our experiments. Another reason may be the different Clostridium strains used by Hurdle et al. (C. difficile BAA-9689, BAA-1803 and BAA-1875 [17]) in comparison to our experiment (non-toxin producing $C$. difficile ATCC 700057). The third possible reason may be the type of reutericyclin used. Chemically, there are two isoforms ((5S)- and (5R)-reutericyclin) and numerous different versions of chemically modified reutericyclins, which have been described in the literature $[16,17]$. In this regard, Hurdle et al. investigated (R)-reutericyclin and its 867 and 1138 modifications [17]. Similarly, Cherian et al. described inhibitory effects against Grampositive bacteria (Enterococcus (En.) faecalis ATCC 33186, Streptococcus (Str.) pyogenes ATCC 700294, Str. pneumonia, Bacillus anthracis sterne, Bacillus subtilis ATCC 23857, C. difficile BAA 1803 and methicillin-susceptible $S$. aureus N315) depending on different modifications of the reutericyclin side chains [16]. It may be possible that the type of isoform ((R) or (S)) affects the antimicrobial activity of reutericyclin. As such, it might be possible that the (S)-isoform of reutericyclin used in this experiment exhibits a reduced antimicrobial activity compared to an $(\mathrm{S}) /(\mathrm{R})$ racemate, the (R)-isoform or other chemically modified versions [16,17] of reutericyclin.

Buffering, treatment with $\mathrm{HCl}$ or proteinase $\mathrm{K}$ or cooking did not influence the activity of (S)-reutericyclin in our study. While lacking effects of buffering or treatment with $\mathrm{HCl}$ or proteinase K are similar to results reported by Messens et al., we could not detect an influence of heat treatment in contrast to their study [14].

Regarding the in vivo application, there are currently no reports regarding the effect of either form of reutericyclin as a pure substance in mice. Despite a possible mitigation of the antimicrobial activity of reutericyclin by cecal contents, effective concentrations should be achievable in the colon $[17,22]$. While there is no such study on reutericyclin as pure substance, there are a few reports investigating the effect of the reutericyclin producing probiotic L. reuteri in piglets $[20,21,23]$. While these animal studies found no effect of reutericyclin on bacterial diversity we encountered a significant decrease of the Bray-Curtis dissimilarity and a trend towards reduced alpha diversity when applying (S)-reutericyclin directly as a postbiotic.

In piglets, administration of the reutericyclin-positive L. reuteri TMW1.656 transiently decreased the abundance of the L. reuteri group in fecal samples in comparison to controls and reduced the proportion of lactobacilli in comparison to the reutericyclin-negative mutant [21]. These results suggest that reutericyclin is a subtle but significant modulator 
of the Lactobacillus community in pigs [21]. In this regard we could demonstrate a tendency towards reduced levels of L. murinus in the (S)-reutericyclin group. In contrast, another study revealed different results regarding bacterial abundance. The authors showed that feed fermentation with $L$. reuteri affected the abundance of few bacterial taxa and particularly reduced the abundance of Enterobacteriaceae when compared to unfermented controls [23]. At the same time, reutericyclin-producing L. reuteri were found to increase the abundance of Dialister spp. and Mitsuokella spp. but did not influence the abundance of clostridial toxins in the feces [23]. Finally, Wang et al. reported that the probiotic-derived antimicrobial compound reutericyclin had only a limited impact on swine intestinal microbiota [20]. All of these investigations deal with probiotic administration of reutericyclin-producing L. reuteri and not with the pure substance itself. At present, it remains unclear if these bacteria produce the (R)-form, (S)-form or a racemate.

While probiotic derived reutericyclin seemed to have a minor effect in swine, mice reacted to (S)-reutericyclin as pure substance with a reduction of potentially beneficial butyrate producers (Roseburia, Eubacterium and Ruminococcus) [24]. Additionally, the increase of Streptococcus has also been encountered in humans-for instance under therapy with proton pump inhibitors [25]. Amongst others, an increase of Streptococcus in the fecal microbiome has been associated with an increased risk of a Clostridium difficile infection in humans [26].

Studies concerning Muribaculum are restricted to a mouse model reporting a decreased relative abundance of Muribaculum in mice developing inflammatory bowel disease [27]. Thus, Muribaculum (amongst others) may be linked to inflammatory processes localized in the intestinal wall. Overall, the application of (S)-reutericyclin caused a decrease of potentially beneficial and an increase of potentially harmful bacteria in our mouse model.

Volatile organic compounds in the headspace of fecal samples are generated during bacterial metabolic processes [28]. VOCs are responsible for the "smell" of different microbial species [29]. Consequently, cultures of some bacteria such as Escherichia coli, Mycobacterium spp., or molds like Aspergillus und Fusarium spp. have distinct VOC profiles allowing recognition of species by their "smell" [30-33]. VOCs within the gut are influenced by intestinal epithelium, the microbiome and diet [28]. In mammals, usually esters dominate the fecal VOC profile as also seen in our measurements. The short carbohydrates pentane and heptane, which were increased under (S)-reutericyclin treatment are substances commonly found in polluted air [34,35]. 2,3-butanedione was decreased in (S)reutericyclin fed mice. It is a plant-growth promoting compound [36] emitted from Bacillus subtilis [37] and has been reported in the headspace of Pseudomonas pseudoalcaligenes [38] and Bacillus mojavensis [36] cultures, but was not previously reported in fecal samples of mice. Among other substances 2-heptanone showed elevations in tissue and fecal samples of dairy cattle and goats infected with Mycobacterium avium subspecies paratuberculosis [39]. In an urinalysis of healthy adult humans, 2-heptanone could be detected among many other VOCs [40]. Furthermore, 2-heptanone was found to decrease in humans subjected to altitude induced hypoxia [41]. In our study, VOC measurements correlated to fecal bacterial abundances to some extent. However, the intestinal microbiome is highly variable throughout the gastrointestinal tract [42] and volatile metabolites are not exclusively produced by certain species. As such, it is almost impossible to directly relate certain VOC substances to specific bacteria in vivo.

One possible limitation of our study is that we only tested one form of reutericyclin ((S)-reutericyclin). Regarding susceptibility testing, this can be justified by the wide variety of reports available for (R)-reutericyclin and its modifications. Additionally, we did not test the susceptibility against other Lactobacillus species. In future studies we plan to elucidate the effect of the different isoforms on the antimicrobial activity against various bacteria including Lactobacillus species of reutericyclin.

In a previous investigation we could demonstrate the variability of the intestinal microbiome throughout the gut, even showing differences between luminal and mucosal microbial compositions [42]. It might well be possible, that animals showed relevant 
alterations at other intestinal levels. However, in this study we limited our microbiome analysis to fecal samples only.

Before initiating this study, it was unclear if the isoform would have an effect on the fecal microbiome of mice at all. It was ethically impossible to include various isoforms and modifications in an animal study without evidence of such an effect. Giving the findings of the present investigation, this is another issue that remains to be addressed in the future.

In conclusion, (S)-reutericyclin had an inhibitory effect on S. epidermidis but exhibited a different antimicrobial activity than described for the (R)-isoform. Moreover, its application in a murine model caused microbial alterations towards dysbiosis.

\section{Materials and Methods}

\subsection{Susceptibility Testing}

Bacteria for resistance testing were obtained from Aurosan $\mathrm{GmbH}$, Essen, Germany. C. difficile (ATCC 700057), Listeria (Lis.) monocytogenes (ATCC 15313), E. coli (ATCC 25922), En. faecium (ATCC 27270), S. aureus (ATCC 29213), S. epidermidis (ATCC 12228), Str. agalactiae (ATCC 13813), P. aeruginosa (ATCC 27853), and Propionibacterium (Pr.) acnes (ATCC 6919) were chosen for bacterial resistance testing. After thawing, bacteria were pre-cultured as broth cultures. Aerobic bacteria were cultivated for $24 \mathrm{~h}$ at $37^{\circ} \mathrm{C}$ and $120 \mathrm{rpm}$ and anaerobic bacteria for $48 \mathrm{~h}$ at $37^{\circ} \mathrm{C}$ and $120 \mathrm{rpm}$. Of each pre-culture, $300 \mu \mathrm{L}$ were then spread on $15 \mathrm{~cm}$ diameter plates with either tryptic soy agar (30 g/L TSB, Fluka Analytical, no T8907-500G, Honeywell, Charlotte, NC, USA; 15 g/L Agar-Agar, Kobe I, no5210-2, Carl Roth GmbH, Karlsruhe, Germany; TSA) for E. coli, S. aureus and P. aeruginosa, TSA with $5 \%$ freshly harvested sheep blood for C. difficile, Str. agalactiae and Pr. acnes, brain heart infusion agar (Brain-Heart-Infusion, no X915.1, 52 g/L, Carl Roth GmbH, Karlsruhe, Germany; 15 g/L Agar-Agar Kobe I, no5210-2, Carl Roth GmbH, Karlsruhe, Germany) for En. faecium and Lis. monocytogenes or nutrient agar $(1 \mathrm{~g} / \mathrm{L}$ beef extract powder, no B488850G, Sigma-Aldrich Handels GmbH, Vienna, Austria; $5 \mathrm{~g} / \mathrm{L}$ peptone, no P0431-250G, Sigma-Aldrich, Handels GmbH, Vienna, Austria; $5 \mathrm{~g} / \mathrm{L} \mathrm{NaCl}$ and 15 or $50 \mathrm{~g} / \mathrm{L}$ AgarAgar) for S. epidermidis. Anaerobic bacteria (C. difficile and Pr. acnes) were cultured for $48 \mathrm{~h}$ in boxes with oxid Anaerogen 2.5 L (Thermo Fisher Scientific, Waldham, MA, USA). The remaining aerobic bacteria grew for $24 \mathrm{~h}$ until a dense bacterial lawn was achieved.

Each microorganism was cultured on five different plates. Using a stencil, 9 disks for resistance testing (BD Sensi-Disc ${ }^{\mathrm{TM}}$, Becton, Dickinson and Company, Franklin Lakes, NJ, USA) were placed on each culture plate using a prepared scheme. (S)-reutericyclin was purchased from BioCrick Biotech Co., Ltd. (Sichuan, China). (S)-reutericyclin was dissolved at a concentration of $62.5 \mu \mathrm{g} / \mathrm{mL}$ in sterile water containing $1 \% \mathrm{H}_{3} \mathrm{PO}_{4}$. Each disk was either treated with respectively $20 \mu \mathrm{L}$ of 1:1 (S)-reutericyclin solution, a 1:2 or 1:4 dilution of the solution, cooked solution $\left(100{ }^{\circ} \mathrm{C}\right.$ for $30 \mathrm{~min}$, no rotation, Thermomixer, HLC, Germany), buffered solution (to $\mathrm{pH} 7$ with $1 \mathrm{n} \mathrm{NaOH}$ ), solution mixed with $1 \mathrm{n} \mathrm{HCl}$ (1:1) or solution treated with $1 \mathrm{mg} / \mathrm{mL}$ Proteinase K (Carl Roth, Germany). Sterile water with $1 \% \mathrm{H}_{3} \mathrm{PO}_{4}$ served as negative control. Either vancomycin (Vancomycin Hikma ${ }^{\circledR}$ $500 \mathrm{mg}$, Hikma Pharma, Planegg, Germany; $0.03 \mathrm{mg} /$ disk) for C. difficile, Str. agalactiae, En. faecium, S. epidermidis, Lis. monocytogenes and S. aureus, or Piperacillin/Tacobactam (PIPeracillin/TAZobactam Kabi 4 g/0.5 g, Fresenius Kabi, Graz, Austria; 0.1 mg/disk) for E. coli, P. aeruginosa and Pr. acnes were used as positive controls. Plates were then incubated at $37^{\circ} \mathrm{C}$ for $24 \mathrm{~h}$ in the case of aerobic and for $48 \mathrm{~h}$ in the case of anaerobic bacteria. Thereafter, plates were photographed and inhibition zones were determined with ImageJ 2.0.0-rc-69/1.52p (ImageJ open source image processing software, http:/ / imagej. net/Contributors, accessed on 12 December 2020).

\subsection{Animal Model}

Male BALBc mice $(n=20)$ were obtained at an age of 7 weeks from the Center for Biomedical Research of the Medical University of Vienna, Austria, as one batch of littermates for microbiome testing. After delivery and an acclimatization period of two 
weeks, mice were split, forming two equal groups ( $n=10$ each) with equal body weight distribution. Mice were kept single-housed in individually ventilated cages under specific pathogen free conditions, a $12 \mathrm{~h}$ light-dark cycle and free access to chow and water at all times. After acclimatization, mice underwent a daily gavage with (S)-reutericyclin as follows: $12.5 \mu \mathrm{g} /$ mouse/day in $400 \mu \mathrm{L}$ sterile water containing $1 \% \mathrm{H}_{3} \mathrm{PO}_{4}$ in the first week to check if mice accepted (S)-reutericyclin without complications. In weeks 2-5, the dosage was increased to $25 \mu \mathrm{g} /$ mouse/day in $400 \mu \mathrm{L}$ sterile water containing $1 \% \mathrm{H}_{3} \mathrm{PO}_{4}$ and in weeks 6-7 to $50 \mu \mathrm{g} / \mathrm{mouse} /$ day in $400 \mu \mathrm{L}$ sterile water containing $1 \% \mathrm{H}_{3} \mathrm{PO}_{4}(n=10$ mice). Sterile water containing $1 \% \mathrm{H}_{3} \mathrm{PO}_{4}$ served as control $(n=10$ mice). After 7 weeks of gavage, mice were euthanized by cranio-cervical dislocation. Two stool samples were collected on the day of euthanasia. One was stored at $-80^{\circ} \mathrm{C}$ until $16 \mathrm{~S}$ based microbiome analysis. The other sample was immediately sent for VOC analysis.

\section{3. $16 S$ Based Microbiome Analysis}

For total DNA isolation, fecal samples were isolated with the Magna Pure LC DNA III Isolation Kit (Bacteria, Fungi) (Roche, Mannheim, Germany) according to published protocols [43]. Briefly, one stool pellet was mixed with $500 \mu \mathrm{L}$ PBS and $250 \mu \mathrm{L}$ bacterial lysis buffer. Samples were homogenized and bead beaten in Magna Lyzer Green bead Tubes (Roche, Mannheim, Germany) in a Magna Lyzer instrument (Roche, Mannheim, Germany) at $6500 \mathrm{rpm}$ for $30 \mathrm{~s}$ two times. Followed by enzymatic lysis with $25 \mu \mathrm{L}$ lysozyme $\left(100 \mathrm{ng} / \mathrm{mL}, 37{ }^{\circ} \mathrm{C}\right.$ for $\left.30 \mathrm{~min}\right)$ and $43.4 \mu \mathrm{L}$ proteinase $\mathrm{K}\left(20 \mathrm{mg} / \mathrm{mL}, 65^{\circ} \mathrm{C}\right.$ for $\left.1 \mathrm{~h}\right)$ samples were heat inactivated at $95{ }^{\circ} \mathrm{C}$ for $10 \mathrm{~min}$ and total DNA was purified in a MagnaPure LC instrument (Roche, Mannheim, Germany) according to manufacturer's instructions. Total DNA was eluted in $100 \mu \mathrm{l}$ elution buffer and stored at $-20^{\circ} \mathrm{C}$ until analysis. For 16S PCR, $2 \mu \mathrm{L}$ of total DNA were used as a template in a $25 \mu \mathrm{L}$ PCR reaction with the FastStart ${ }^{\mathrm{TM}}$ (Sigma Aldrich Handels GesmbH, Vienna, Austria) High Fidelity PCR-System (Sigma, Darmstadt, Germany) according to the manufacturer's instructions and the target specific primers 515F (5'-GTGYCAGCMGCCGCGGTAA- $\left.3^{\prime}\right)$ and 806R (5'GGACTACNVGGGTWTCTAAT-3') for 30 cycles in triplicates. Triplicates were pooled, normalized, indexed and purified according to published protocols [43]. The final pool was sequenced on an Illumina MiSeq desktop sequencer at 9 pM and v 3600 cycles chemistry. FASTQ raw files were used for data analysis.

A total of 2,479,083 MiSeq paired end FASTQ reads were used for further analysis. The DADA2 pipeline for modeling and correcting Illumina-sequenced amplicon errors for quality-filtering [44] was used with standard settings for denoising, dereplicating, merging and check for chimeras as implemented in QIIME2 2018.4 microbiome bioinformatics platform [45]. QIIME2 was integrated in our own non-public instance of Galaxy (MedBioNode https: / / galaxy.medunigraz.at accessed on 12 August 2020) [46]. Taxonomic assignment of the DADA2 representative sequences was provided with the QIIME2 sklearn-based classifier against SILVA rRNA database release 132 at $99 \%$ identity [47]. To interpret and compare taxonomic information, $16 \mathrm{~S}$ rRNA data was transferred to the Calypso online software (Calypso $8.84^{\circledR}$, accessible through http:/ / cgenome.net/wiki/index.php/Calypso, last accessed 24 August 2020) [48]. Samples were rarefied to a read depth of 12,774. Alpha diversity was calculated using Chao1 estimator, Inversed Simpson and Shannon index. Relative abundances (total sum scaling with square root transformation) were used for further group comparisons. Beta diversity was examined using a redundancy analysis (RDA) and colored principal component analysis plots (PCoA) based on Bray-Curtis dissimilarity score. The identification of discriminating taxa between the groups was performed with a linear discriminant effect size (LEfSe) analysis. Differentially abundant taxa identified by LEfSe analysis were considered relevant if the differences between groups could be verified by ANOVA $(p<0.1)$. 


\subsection{Stool VOC Analysis}

Samples were weighed and stored in glass vials (Gerstel GmbH, Germany) and stored at $6{ }^{\circ} \mathrm{C}$. Room air samples were collected at the same time points to correct for possible contamination. All samples were immediately sent to the partner via overnight express for gas chromatography/mass spectrometry (GC-MS). VOC analysis was performed in the headspace of samples as previously reported [49-51]. VOCs were preconcentrated with a commercially available solid phase micro extraction (SPME) fiber (carboxen/polymethylsiloxane, Supelco, Bellefonte, PA, USA). An Agilent 7890 A gas chromatograph (GC) coupled to an Agilent 5975 C inert XL mass selective detector (MSD) was used to separate and identify the VOCs desorbed from the SPME device. Detected marker substances were identified tentatively from a mass spectral library (National Institute of Standards and Technology 2005; NIST 2005, Gatesburg, PA, USA) and by retention time matching. Results were corrected for the stool weight. When the mean of the room air samples exceeded $30 \%$ of the mean of the headspace samples, a possible contamination was recorded and the substance was excluded from further analysis. The responses of a selected $\mathrm{m} / \mathrm{q}$ ratio at a defined retention time for each substance were recorded, integrated and used for group comparison.

\subsection{Statistics}

Data was managed with Microsoft Excel 2016 ${ }^{\circledR}$. For statistical analysis, data was transferred to SPSS $26.0^{\circledR}$. Graphical work-up was performed with GraphPad Prism $9^{\circledR}$. The Heatmap was drawn with the heatmap function of ggplot2 package (version 3.3.3) for RStudio $^{\circledR}$ (version 1.4.1106). Metric data is displayed as median and interquartile range (IQR). A Mann-Whitney U-Test was used to determine group differences. Correlation analyses were conducted between bacteria resulting from LEfSe analysis and VOCS with group differences $p<0.1$ using the corrplot package (version 0.84 ) for RStudio ${ }^{\circledR}$ applying a Spearman-Rho Test. $P$-values $<0.05$ were considered statistically significant.

Author Contributions: Conceptualization C.C., G.S. and R.G.; methodology, B.O., A.H., S.M., S.H., P.F. and I.K.; validation, S.H., W.M., V.S. and R.G.; formal analysis, I.K., P.F., A.H., S.H., S.M. and B.O.; resources, C.C.; writing-original draft preparation, B.K.; writing-review and editing, all authors listed; visualization, B.O. and A.H.; supervision, C.C.; funding acquisition, C.C. All authors have read and agreed to the published version of the manuscript.

Funding: This research was funded by The Österreichische Forschungsförderungsgesellschaft (FFG), grant number 867991.

Institutional Review Board Statement: All animal experiments were approved by the veterinary board (BMBWF-66.010/0153-V/3b/2019).

Informed Consent Statement: Not applicable.

Data Availability Statement: Data from microbiome and VOC analysis is available from a Mendeley Data ${ }^{\circledR}$ repository (access: http:/ / dx.doi.org/10.17632/xzb8wzxt5z.1).

Acknowledgments: We would like to extend our special gratitude to Wolfgang Stadlbauer who helped us with the chemical understanding of reutericyclin isoforms.

Conflicts of Interest: The authors declare no conflict of interest. The funder had no role in the design of the study; in the collection, analyses, or interpretation of data; in the writing of the manuscript, or in the decision to publish the results.

\section{References}

1. Angelakis, E.; Merhej, V.; Raoult, D. Related actions of probiotics and antibiotics on gut microbiota and weight modification. Lancet Infect. Dis. 2013, 13, 889-899. [CrossRef]

2. Cani, P.D.; Amar, J.; Iglesias, M.A.; Poggi, M.; Knauf, C.; Bastelica, D.; Neyrinck, A.M.; Fava, F.; Tuohy, K.M.; Chabo, C.; et al. Metabolic endotoxemia initiates obesity and insulin resistance. Diabetes 2007, 56, 1761-1772. [CrossRef] 
3. Devkota, S.; Wang, Y.; Musch, M.W.; Leone, V.; Fehlner-Peach, H.; Nadimpalli, A.; Antonopoulos, D.A.; Jabri, B.; Chang, E.B. Dietary-fat-induced taurocholic acid promotes pathobiont expansion and colitis in Il10-/- mice. Nature 2012, 487, 104-108. [CrossRef]

4. Koeth, R.A.; Wang, Z.; Levison, B.S.; Buffa, J.A.; Org, E.; Sheehy, B.T.; Britt, E.B.; Fu, X.; Wu, Y.; Li, L.; et al. Intestinal microbiota metabolism of L-carnitine, a nutrient in red meat, promotes atherosclerosis. Nat. Med. 2013, 19, 576-585. [CrossRef]

5. Spencer, M.D.; Hamp, T.J.; Reid, R.W.; Fischer, L.M.; Zeisel, S.H.; Fodor, A.A. Association between composition of the human gastrointestinal microbiome and development of fatty liver with choline deficiency. Gastroenterology 2011, 140, 976-986. [CrossRef]

6. Redman, M.G.; Ward, E.J.; Phillips, R.S. The efficacy and safety of probiotics in people with cancer: A systematic review. Ann. Oncol. 2014, 25, 1919-1929. [CrossRef]

7. De Weirdt, R.; Crabbe, A.; Roos, S.; Vollenweider, S.; Lacroix, C.; van Pijkeren, J.P.; Britton, R.A.; Sarker, S.; Van de Wiele, T.; Nickerson, C.A. Glycerol supplementation enhances L. reuteri's protective effect against S. Typhimurium colonization in a 3-D model of colonic epithelium. PLoS ONE 2012, 7, e37116. [CrossRef]

8. Montiel, R.; Martin-Cabrejas, I.; Langa, S.; El Aouad, N.; Arques, J.L.; Reyes, F.; Medina, M. Antimicrobial activity of reuterin produced by Lactobacillus reuteri on Listeria monocytogenes in cold-smoked salmon. Food Microbiol. 2014, 44, 1-5. [CrossRef]

9. Ganzle, M.G. Reutericyclin: Biological activity, mode of action, and potential applications. Appl. Microbiol. Biotechnol. 2004, 64, 326-332. [CrossRef]

10. Ganzle, M.G.; Holtzel, A.; Walter, J.; Jung, G.; Hammes, W.P. Characterization of reutericyclin produced by Lactobacillus reuteri LTH2584. Appl. Environ. Microbiol. 2000, 66, 4325-4333. [CrossRef]

11. Lin, X.B.; Lohans, C.T.; Duar, R.; Zheng, J.; Vederas, J.C.; Walter, J.; Ganzle, M. Genetic determinants of reutericyclin biosynthesis in Lactobacillus reuteri. Appl. Environ. Microbiol. 2015, 81, 2032-2041. [CrossRef]

12. Holtzel, A.; Ganzle, M.G.; Nicholson, G.J.; Hammes, W.P.; Jung, G. The First Low Molecular Weight Antibiotic from Lactic Acid Bacteria: Reutericyclin, a New Tetramic Acid. Angew. Chem. Int. Ed. Engl. 2000, 39, 2766-2768. [CrossRef]

13. Schobert, R. Domino syntheses of bioactive tetronic and tetramic acids. Naturwissenschaften 2007, 94, 1-11. [CrossRef]

14. Messens, W.; De Vuyst, L. Inhibitory substances produced by Lactobacilli isolated from sourdoughs-A review. Int. J. Food Microbiol. 2002, 72, 31-43. [CrossRef]

15. Ganzle, M.G.; Vogel, R.F. Studies on the mode of action of reutericyclin. Appl. Environ. Microbiol. 2003, 69, 1305-1307. [CrossRef]

16. Cherian, P.T.; Wu, X.; Maddox, M.M.; Singh, A.P.; Lee, R.E.; Hurdle, J.G. Chemical modulation of the biological activity of reutericyclin: A membrane-active antibiotic from Lactobacillus reuteri. Sci. Rep. 2014, 4, 4721. [CrossRef]

17. Hurdle, J.G.; Heathcott, A.E.; Yang, L.; Yan, B.; Lee, R.E. Reutericyclin and related analogues kill stationary phase Clostridium difficile at achievable colonic concentrations. J. Antimicrob. Chemother. 2011, 66, 1773-1776. [CrossRef]

18. Yendapally, R.; Hurdle, J.G.; Carson, E.I.; Lee, R.B.; Lee, R.E. N-substituted 3-acetyltetramic acid derivatives as antibacterial agents. J. Med. Chem. 2008, 51, 1487-1491. [CrossRef]

19. Casas, I.A.; Dobrogosz, W.J. Lactobacillus reuteri: An overview of a new probiotic for humans and animals. Microecol. Ther. 1997, $25,221-231$.

20. Wang, W.; Zijlstra, R.T.; Ganzle, M.G. Feeding Limosilactobacillus fermentum K9-2 and Lacticaseibacillus casei K9-1, or Limosi Lactobacillus reuteri TMW1.656 Reduces Pathogen Load in Weanling Pigs. Front. Microbiol. 2020, 11, 608293. [CrossRef]

21. Zhao, X.; Wang, W.; Blaine, A.; Kane, S.T.; Zijlstra, R.T.; Ganzle, M.G. Impact of probiotic Lactobacillus sp. on autochthonous lactobacilli in weaned piglets. J. Appl. Microbiol. 2019, 126, 242-254. [CrossRef]

22. $\mathrm{Wu}, \mathrm{X}$; Cherian, P.T.; Lee, R.E.; Hurdle, J.G. The membrane as a target for controlling hypervirulent Clostridium difficile infections. J. Antimicrob. Chemother. 2013, 68, 806-815. [CrossRef]

23. Yang, Y.; Zhao, X.; Le, M.H.; Zijlstra, R.T.; Ganzle, M.G. Reutericyclin producing Lactobacillus reuteri modulates development of fecal microbiota in weanling pigs. Front. Microbiol. 2015, 6, 762. [CrossRef]

24. Riviere, A.; Selak, M.; Lantin, D.; Leroy, F.; De Vuyst, L. Bifidobacteria and Butyrate-Producing Colon Bacteria: Importance and Strategies for Their Stimulation in the Human Gut. Front. Microbiol. 2016, 7, 979. [CrossRef] [PubMed]

25. Imhann, F.; Bonder, M.J.; Vich Vila, A.; Fu, J.; Mujagic, Z.; Vork, L.; Tigchelaar, E.F.; Jankipersadsing, S.A.; Cenit, M.C.; Harmsen, H.J.; et al. Proton pump inhibitors affect the gut microbiome. Gut 2016, 65, 740-748. [CrossRef]

26. Freedberg, D.E.; Toussaint, N.C.; Chen, S.P.; Ratner, A.J.; Whittier, S.; Wang, T.C.; Wang, H.H.; Abrams, J.A. Proton Pump Inhibitors Alter Specific Taxa in the Human Gastrointestinal Microbiome: A Crossover Trial. Gastroenterology 2015, 149, 883-885.e889. [CrossRef]

27. Dobranowski, P.A.; Tang, C.; Sauve, J.P.; Menzies, S.C.; Sly, L.M. Compositional changes to the ileal microbiome precede the onset of spontaneous ileitis in SHIP deficient mice. Gut Microbes 2019, 10, 578-598. [CrossRef] [PubMed]

28. Ahmed, I.; Greenwood, R.; Costello Bde, L.; Ratcliffe, N.M.; Probert, C.S. An investigation of fecal volatile organic metabolites in irritable bowel syndrome. PLoS ONE 2013, 8, e58204. [CrossRef] [PubMed]

29. Schulz, S.; Dickschat, J.S. Bacterial volatiles: The smell of small organisms. Nat. Prod. Rep. 2007, 24, 814-842. [CrossRef]

30. Maddula, S.; Blank, L.M.; Schmid, A.; Baumbach, J.I. Detection of volatile metabolites of Escherichia coli by multi capillary column coupled ion mobility spectrometry. Anal. Bioanal. Chem. 2009, 394, 791-800. [CrossRef]

31. Syhre, M.; Chambers, S.T. The scent of Mycobacterium tuberculosis. Tuberculosis 2008, 88, 317-323. [CrossRef] [PubMed]

32. Syhre, M.; Scotter, J.M.; Chambers, S.T. Investigation into the production of 2-Pentylfuran by Aspergillus fumigatus and other respiratory pathogens in vitro and human breath samples. Med. Mycol. 2008, 46, 209-215. [CrossRef] 
33. Trefz, P.; Koehler, H.; Klepik, K.; Moebius, P.; Reinhold, P.; Schubert, J.K.; Miekisch, W. Volatile emissions from Mycobacterium avium subsp. paratuberculosis mirror bacterial growth and enable distinction of different strains. PLOS ONE 2013, 8, e76868. [CrossRef]

34. Lv, D.; Lu, S.; Tan, X.; Shao, M.; Xie, S.; Wang, L. Source profiles, emission factors and associated contributions to secondary pollution of volatile organic compounds (VOCs) emitted from a local petroleum refinery in Shandong. Environ. Pollut. 2021, 274, 116589. [CrossRef] [PubMed]

35. Pitiriciu, M.; Tansel, B. Volatile organic contaminants (VOCs) emitted from sewer networks during wastewater collection and transport. J. Environ. Manag. 2021, 285, 112136. [CrossRef] [PubMed]

36. Rath, M.; Mitchell, T.R.; Gold, S.E. Volatiles produced by Bacillus mojavensis RRC101 act as plant growth modulators and are strongly culture-dependent. Microbiol. Res. 2018, 208, 76-84. [CrossRef]

37. Park, H.B.; Lee, B.; Kloepper, J.W.; Ryu, C.M. One shot-two pathogens blocked: Exposure of Arabidopsis to hexadecane, a long chain volatile organic compound, confers induced resistance against both Pectobacterium carotovorum and Pseudomonas syringae. Plant Signal. Behav. 2013, 8, e24619. [CrossRef]

38. Yasmin, H.; Rashid, U.; Hassan, M.N.; Nosheen, A.; Naz, R.; Ilyas, N.; Sajjad, M.; Azmat, A.; Alyemeni, M.N. Volatile organic compounds produced by Pseudomonas pseudoalcaligenes alleviated drought stress by modulating defense system in maize (Zea mays L.). Physiol. Plant 2020. [CrossRef]

39. Vitense, P.; Kasbohm, E.; Klassen, A.; Gierschner, P.; Trefz, P.; Weber, M.; Miekisch, W.; Schubert, J.K.; Mobius, P.; Reinhold, P.; et al. Detection of Mycobacterium avium ssp. paratuberculosis in Cultures from Fecal and Tissue Samples Using VOC Analysis and Machine Learning Tools. Front Vet. Sci. 2021, 8, 620327. [CrossRef]

40. Mochalski, P.; Unterkofler, K. Quantification of selected volatile organic compounds in human urine by gas chromatography selective reagent ionization time of flight mass spectrometry (GC-SRI-TOF-MS) coupled with head-space solid-phase microextraction (HS-SPME). Analyst 2016, 141, 4796-4803. [CrossRef]

41. Harshman, S.W.; Geier, B.A.; Fan, M.; Rinehardt, S.; Watts, B.S.; Drummond, L.A.; Preti, G.; Phillips, J.B.; Ott, D.K.; Grigsby, C.C. The identification of hypoxia biomarkers from exhaled breath under normobaric conditions. J. Breath Res. 2015, 9, 047103. [CrossRef]

42. Klymiuk, I.; Singer, G.; Castellani, C.; Trajanoski, S.; Obermuller, B.; Till, H. Characterization of the Luminal and MucosaAssociated Microbiome along the Gastrointestinal Tract: Results from Surgically Treated Preterm Infants and a Murine Model. Nutrients 2021, 13, 1030. [CrossRef]

43. Klymiuk, I.; Bilgilier, C.; Stadlmann, A.; Thannesberger, J.; Kastner, M.T.; Hogenauer, C.; Puspok, A.; Biowski-Frotz, S.; SchrutkaKolbl, C.; Thallinger, G.G.; et al. The Human Gastric Microbiome Is Predicated upon Infection with Helicobacter pylori. Front. Microbiol. 2017, 8, 2508. [CrossRef]

44. Callahan, B.J.; McMurdie, P.J.; Rosen, M.J.; Han, A.W.; Johnson, A.J.; Holmes, S.P. DADA2: High-resolution sample inference from Illumina amplicon data. Nat. Methods 2016, 13, 581-583. [CrossRef]

45. Bolyen, E.; Rideout, J.R.; Dillon, M.R.; Bokulich, N.A.; Abnet, C.C.; Al-Ghalith, G.A.; Alexander, H.; Alm, E.J.; Arumugam, M.; Asnicar, F.; et al. Reproducible, interactive, scalable and extensible microbiome data science using QIIME 2. Nat. Biotechnol. 2019, 37, 852-857. [CrossRef]

46. Afgan, E.; Baker, D.; Batut, B.; van den Beek, M.; Bouvier, D.; Cech, M.; Chilton, J.; Clements, D.; Coraor, N.; Gruning, B.A.; et al. The Galaxy platform for accessible, reproducible and collaborative biomedical analyses: 2018 update. Nucleic Acids Res. 2018, 46, W537-W544. [CrossRef] [PubMed]

47. Quast, C.; Pruesse, E.; Yilmaz, P.; Gerken, J.; Schweer, T.; Yarza, P.; Peplies, J.; Glockner, F.O. The SILVA ribosomal RNA gene database project: Improved data processing and web-based tools. Nucleic Acids Res. 2013, 41, D590-D596. [CrossRef] [PubMed]

48. Zakrzewski, M.; Proietti, C.; Ellis, J.J.; Hasan, S.; Brion, M.J.; Berger, B.; Krause, L. Calypso: A user-friendly web-server for mining and visualizing microbiome-environment interactions. Bioinformatics 2017, 33, 782-783. [CrossRef] [PubMed]

49. Bergmann, A.; Trefz, P.; Fischer, S.; Klepik, K.; Walter, G.; Steffens, M.; Ziller, M.; Schubert, J.K.; Reinhold, P.; Kohler, H.; et al. In Vivo Volatile Organic Compound Signatures of Mycobacterium avium subsp. paratuberculosis. PLoS ONE 2015, 10, e0123980. [CrossRef]

50. Miekisch, W.; Trefz, P.; Bergmann, A.; Schubert, J.K. Microextraction techniques in breath biomarker analysis. Bioanalysis 2014, 6, 1275-1291. [CrossRef]

51. Obermuller, B.; Singer, G.; Kienesberger, B.; Klymiuk, I.; Sperl, D.; Stadlbauer, V.; Horvath, A.; Miekisch, W.; Gierschner, P.; Grabherr, R.; et al. The Effects of Prebiotic Supplementation with OMNi-LOGiC ${ }^{\circledR}$ FIBRE on Fecal Microbiome, Fecal Volatile Organic Compounds, and Gut Permeability in Murine Neuroblastoma-Induced Tumor-Associated Cachexia. Nutrients 2020, 12, 2029. [CrossRef] [PubMed] 